\title{
Polyphenol and Biogenic Amine Profiles of Albana and Lambrusco Grape Berries and Wines Obtained Following Different Agricultural and Oenological Practices
}

\author{
Annalisa Tassoni, Nunzio Tango, Maura Ferri \\ Department of Biological, Geological and Environmental Sciences University of Bologna, Bologna, Italy. \\ Email: annalisa.tassoni2@unibo.it
}

Received November $6^{\text {th }}, 2013$; revised December $6^{\text {th }}, 2013$; accepted December $13^{\text {th }}$, 2013

Copyright @ 2014 Annalisa Tassoni et al. This is an open access article distributed under the Creative Commons Attribution License, which permits unrestricted use, distribution, and reproduction in any medium, provided the original work is properly cited. In accordance of the Creative Commons Attribution License all Copyrights (c) 2014 are reserved for SCIRP and the owner of the intellectual property Annalisa Tassoni et al. All Copyright (C) 2014 are guarded by law and by SCIRP as a guardian.

\section{ABSTRACT}

The levels of polyphenols, anthocyanins, antioxidant activity and of biogenic amines, were measured in white (Albana) and red (Lambrusco) grape berries and wines from the Emilia-Romagna region (Italy) obtained following conventional, organic and biodynamic agricultural and oenological practices. No significant difference was shown among the samples coming from different agricultural and winemaking practices, with few exceptions of single compounds. Biogenic amine amounts were higher in red than in white berries and wines. Putrescine and histamine were the most abundant biogenic amines respectively in berries and wines of both cultivars. Red grapes and wines were richer in anthocyanins and showed higher antioxidant activity than white ones. The total level of polyphenols was similar in red and white berries and wines, but with different metabolite profiles depending on the grape variety.

\section{KEYWORDS}

Antioxidant Activity; Biogenic Amines; Polyphenols; Polyamines; Vitis Vinifera

\section{Introduction}

The bio-active nutraceutical and anti-nutraceutical compounds present in food and beverages have great potential influence on human health, however the molecular composition and complexity of many foods cannot as yet be fully described. Many studies have been recently performed regarding food metabolomics and the results evidenced that generally the amount and spectrum of nutrients and metabolites in food and beverages not only depends upon their processing and storage methods but also is largely influenced by the farming system with which the raw materials are produced. Several published papers aimed to compare the metabolite profile of crops grown under conventional, organic and biodynamic agricultural practices. In general, organic products are perceived by the public as healthier and safer than those produced through conventional agriculture. There are fundamental differences in organic and conventional production practices, but limited information is available on how these influence the nutritional quality of food. Research data showed that some crops grown under organic farming practices contained more bioactive substances such as flavones, vitamin C, carotenoids and total polyphenols [1]. Some studies confirmed better biological activity of organic products versus conventional due to the higher content of bioactive compounds [1,2]. Conversely, other researches evidenced no significant difference between general metabolic profile, phenolic levels and nutritional values of buckwheat groats [3], wheat grains [4] and apples [5] grown under conventional and organic farming. Biodynamic farming is similar in many ways to better-known organic agriculture. Both use composting and cover cropping instead of mineral fertilizing and ban pesticides, herbicides, hormones and other chemicals. The difference from organic agriculture lies in the use of biodynamic preparations which contain specific herbs or minerals, treated or fermented with animal 
organs and applied in homoeopathic form generally as field sprays after dynamisation. The aims of biodynamic preparations have been described and may to lie in the improvement of soil and crop quality [6]. Two studies on grape quality showed no differences between metabolic profiles (nutraceutical and anti-nutraceutical compounds) of conventionally, organically and biodynamically-cultivated grapes and wines [6,7]. Among nutraceutical compounds polyphenols, such as flavonoids (e.g. catechins and anthocyanins) and stilbenes (e.g. resveratrol (RESV)), have been widely investigated in grape and wines given their well ascertained health-protective and antioxidant properties [8,9]. Polyphenols are one of the most widespread groups of plant metabolites occurring both as glycosides and aglycones [10]. After ingestion through daily diet, they are absorbed by the small intestine mucosa increasing the antioxidant capacity of blood and aiding in the prevention of cancer and cardiovascular diseases [8,11]. Great attention has been given to the stilbene family and in particular to RESV and to its [11] mono-glucosylated derivatives piceid (PIC) and resveratroloside (RDE). PIC and RDE are present at high levels in grape berries and wines and possess antioxidant activity comparable to free RESV but, due to the presence of the glucose residue, have a more extended half-life and bioavailability [12]. In addition piceatannol (PICEAT) is a naturally occurring derivative of RESV synthesized in grape berries only during ripening [13] and was shown to inhibit the proliferation of cancer cell lines via apoptosis and cell cycle arrest [11]. Among the anti-nutraceutical compounds, amines are basic nitrogenous compounds generally synthesized by metabolic pathways that usually involve decarboxylation of precursor amino acids [14,15]. The term "biogenic amines" defines decarboxylation products such as histamine (HIM), serotonine, tyramine (TYM), tryptamine (TRYPT), phenylethylamine but also comprehends aliphatic polyamines such as agmatine, putrescine (PUT), cadaverine (CAD), spermidine (SPD) and spermine (SPM). In food and beverages, biogenic amines are formed by the enzymes from raw material or are generated by microbial decarboxylation of amino acids and in particular they are present in fermented foods such as cheese, wine, beer, sauerkrauts [16]. Some types of biogenic amines (such as HIM, TYM, TRYPT, PUT and CAD) are undesirable in all food and beverages because, if absorbed at too high concentrations, they may cause headaches, respiratory distress, heart palpitation, hypertension or hypotension, and several allergenic disorders [16]. Aliphatic polyamines, such as PUT, SPD and SPM, are essential for normal cell growth but also, at high concentrations, may sustain cancer cell proliferation [16]. Several investigations reported the presence of more than twenty amines in wines and their total concentration has been reported to range from a few to about 50
mg/L, depending on many factors including winemaking conditions, must fermentation and aging. HIM, TYM and PUT are the most significant biogenic amines found in wines $[15,16]$.

The public opinion generally considers organic and biodynamic foods healthier than the correspondent conventional ones, however the scientific evidences are still poor and ambiguous. In this view, the present study aims to compare two types of conventional, organic and biodynamic Italian white and red grapes and the related wines, to ascertain whether the different agricultural practices and winemaking procedures, may directly influence the profiles and contents of polyphenols and biogenic amines and the antioxidant capacity.

\section{Materials and Methods}

\subsection{Materials}

Grape berries of Vitis vinifera var. Albana (white) and Lambrusco Grasparossa (red), both autochthonous varieties of the Emilia-Romagna region (Italy), and the derived wines were collected in 2009 from local producers. Albana and Lambrusco grapevines were grown by using the following conventional, organic and biodynamic agricultural practices (Albana from Azienda Agricola Rambaldi, Ozzano, Bologna and Lambrusco from Azienda Agricola Mauro Pallotti, Castelfranco Emilia, Modena). The berries (white or red) were harvested during vintage time on the same day, picking bunches from different plants grown in different vineyard areas, and at different light/shadow exposure. About $10 \mathrm{~kg}$ of grape were collected for each vineyard, immediately frozen with liquid nitrogen and stored at $-80^{\circ} \mathrm{C}$. The grapes were successively ground in liquid nitrogen and the powders, stored at $-80^{\circ} \mathrm{C}$, were used for the following analyses. Wines were produced from grapes on site by the same producers/wineries according to the relative conventional, organic and biodynamic technical regulations. Unfortunately given to production technical problems, it was not possible to collect and analyse the conventional Lambrusco wine. The wines were collected from wineries at the end of the production process, immediately after bottling, centrifuged at $13,000 \times \mathrm{g}$ for $10 \mathrm{~min}$ to remove solid residues and immediately stored at $-20^{\circ} \mathrm{C}$ until analysis.

\subsection{Total Polyphenol Quantification}

Total polyphenols were determined by using the FolinCiocalteu method [17]. Grape powder samples (0.5 g) were extracted by overnight shaking at $4{ }^{\circ} \mathrm{C}$ with $4 \mathrm{~mL}$ of 98:2 methanol: $12 \mathrm{~N} \mathrm{HCl}$ and centrifuged $5000 \times \mathrm{g}$ for 15 min at $4^{\circ} \mathrm{C}$. A suitable volume of grape methanolic extracts or of wines was diluted to $1.6 \mathrm{~mL}$ with water and 
$100 \mu \mathrm{L}$ of Folin-Ciocalteu reagent were added. After 5 min the reaction was stopped with $300 \mu \mathrm{L}$ of $20 \%$ (w/v) sodium carbonate. The mixture was vortexed for $15 \mathrm{sec}$ and incubated at $40^{\circ} \mathrm{C}$ for $30 \mathrm{~min}$ in the dark, before measuring the absorbance at $765 \mathrm{~nm}$. The results were expressed as gallic acid (GA) equivalents by means of a calibration curve.

\subsection{Quantification of Total Anthocyanins}

Anthocyanins were extracted from grape powders $(0.5 \mathrm{~g})$ which were resuspended in $4 \mathrm{~mL}$ of extraction solution (98:2 methanol: $12 \mathrm{~N} \mathrm{HCl}$ ) and incubated at $65^{\circ} \mathrm{C}$ for $2 \mathrm{~h}$. After centrifugation for $10 \mathrm{~min}$ at $4500 \times \mathrm{g}$ at room temperature, suitable volumes of supernatants (grape extracts) and of wines were used for spectrophotometric analyses. Absorbance (Abs) was measured for each sample at 530 and $657 \mathrm{~nm}$ and the anthocyanin absorbance was calculated as $\Delta \mathrm{Abs}$ anthocyanins $=\mathrm{Abs}_{530}-\left(0.25 \cdot \mathrm{Abs}_{657}\right) . \mathrm{Abs}_{657}$ was used to correct for the presence of chlorophyll degradation products such as pheophytins [18].

\subsection{Determination of Antioxidant Activity by DPPH Method}

Antioxidant activity was measured using the method based on the DPPH (2,2-diphenyl-1-picrylhydrazyl) radical scavenging capacity [19], with minor modifications. Aliquots of ascorbic acid (AA) standard solution, grape methanolic extracts (see paragraph 2.2) or wine samples, were added to $0.5 \mathrm{~mL}$ of $90 \mu \mathrm{M}$ DPPH solution (dissolved in methanol) and the total reaction volume was taken up to $1 \mathrm{~mL}$ with $95 \%(\mathrm{v} / \mathrm{v})$ methanol. The mixture was vortexed for $15 \mathrm{sec}$ and left to stand at room temperature for $30 \mathrm{~min}$ in the dark, before measuring the absorbance at $517 \mathrm{~nm}$. The results were expressed as AA equivalents by means of the dose-response calibration curve.

\subsection{Quantification of Polyphenols by HPLC}

Polyphenols were extracted from about $0.5 \mathrm{~g}$ of grape powders (incubated overnight with $5 \mathrm{~mL}$ of $95 \%(\mathrm{v} / \mathrm{v})$ methanol) and from $5 \mathrm{~mL}$ of wines. The samples were loaded onto a Strata-X column (33 mm polymeric sorbent $60 \mathrm{mg}$ in $3 \mathrm{~mL}$, Phenomenex, Torrence, CA, USA) and polyphenols were eluted by $100 \%(\mathrm{v} / \mathrm{v})$ methanol, completely dried and resuspended in $200 \mu \mathrm{L}$ of 1:9 ACN: $0.2 \%(\mathrm{v} / \mathrm{v})$ acetic acid before being directly injected into the HPLC-DAD (column Gemini C18, $5 \mu \mathrm{m}$ particles $250 \times 4.6 \mathrm{~mm}$, pre-column SecurityGuard Ea, Phenomenex, Torrence CA, USA) equipped with an on-line diode array detector (MD-2010, Plus, Jasco Instruments, Großumstad, Germany), as described by Ferri et al. [18]. The adopted HPLC-diode array detector (DAD) separation procedure allowed the acquisition of chromatogram spectra from 200 to $700 \mathrm{~nm}$ and the simultaneous analysis of the following compounds grouped according to their maximum wavelength detection peak: gallic acid (GA), vanillin (VAN), vanillic acid (AVAN), (+)-catechin (CAT), (-)-epicatechin (EC), (-)-epigallocatechin-gallate (EGCG), epigallocatechin (EGC), epicatechin-3-gallate (ECG), at $270 \mathrm{~nm}$; cis-piceid (CPIC), cis-resveratrol (CRESV), naringenin (NAR) at $285 \mathrm{~nm}$; trans-resveratroloside (TRDE), trans-piceid (TPIC) trans-resveratrol (TRESV) at $305 \mathrm{~nm}$; cis-resveratroloside (CRDE), piceatannol (PICEAT) at 323nm; quercetin (QUERC), rutin (RUT) and myricetin (MYR) at 365nm. The HPLC standards were purchased from Sigma-Aldrich (Milano, Italy) except for cis-RESV, trans- and cis-RDE, trans- and cis-PIC which were obtained as reported by Ferri et al. [18].

\subsection{Quantification of Biogenic Amines}

Free biogenic amines tryptamine (TRYPT), histamine (HIM), tyramine (TYM), diamine-propane (DAP), cadaverine (CAD), putrescine (PUT), spermidine (SPD) and spermine (SPM)) analyses were performed [20]. All the standard compounds were purchased from Sigma-Aldrich (Milano, Italy). The grape samples (about $0.2 \mathrm{~g}$ of powders) were homogenised in 10 volumes of $4 \%(\mathrm{v} / \mathrm{v})$ cold perchloric acid and centrifuged at 20,000 $\times \mathrm{g}$ for $30 \mathrm{~min}$ at $4^{\circ} \mathrm{C}$ and the supernatant was used for free amine determination. Aliquots $(0.2 \mathrm{~mL})$ of supernatants or of wines were derivatised with dansyl-chloride $(3 \mathrm{mg} / \mathrm{mL}$ of acetone), extracted with toluene and analysed by high-performance liquid chromatography (HPLC, Jasco, Großumstad, Germany; equipped with an on-line spectrofluorometer Jasco 821-FP; excitation wavelength $360 \mathrm{~nm}$, emission wavelength $510 \mathrm{~nm}$ ) with a reverse phase C18 column (Gemini, $5 \mu \mathrm{M}$ particle diameter, $4.6 \times 250 \mathrm{~mm}$, Phenomenex, Torrance, CA, USA) and the solvent gradient $(1 \mathrm{~mL} / \mathrm{min})$ was as described in [7].

\subsection{Statistical Analyses}

Two independent replicates were performed for all experiments and the relative extracts were analysed in technical duplicates. The presented results are the means of the four data $(n=4) \pm$ SE. The significance of the data was analysed by using the Student's $t$-test $(p<0.05)$ among each different group of white and red berry or wine samples.

\section{Results}

\subsection{Grape Berries}

Total polyphenol and anthocyanin amounts and antioxi- 
dant activity (Table 1) were determined in Albana and Lambrusco berries by using spectrophotometric methods. Total polyphenols resulted to be on average 4.6 and $8.2 \mathrm{~g}$ of gallic acid (GA) equivalents/kg of fresh weight (g GA eq/kgFW) respectively for Albana and Lambrusco berries with a double amount in red compared to white berries and no significant difference among the three agricultural practices in both cultivars. As expected the levels of total anthocyanins were much higher in red than in white berries (about 320-fold for conventional and on average 210-fold for organic and biodynamic samples) (Table 1). The antioxidant activity of the different grape berries was measured by using the DPPH method. On average Albana berries showed a 3.5-fold lower antioxidant capacity compared to Lambrusco ones (in agreement with the levels of total anthocyanins), with $A B$ and LB having the highest activity respectively for white and red samples (Table 1 ).

The detailed polyphenolic profile was determined by HPLC-DAD. Several compounds were detected, among which the most relevant were catechins (Figure 1(a)) and stilbenes (Figure 1(b)). In general total catechins were 22 to 7 -fold higher than total stilbenes, with LC (showing very high levels of epigallo-catechin, EGC) and $\mathrm{AO}$ having respectively the minimum and maximum difference (Figures 1(a) and (b)). A different spectrum of catechins and stilbenes was detected in white and red grapes but not in berries of the same cultivar grown following different agricultural methods. Epicatechin (EC) and catechin (CAT) (EGC in LC) were the catechins most abundant respectively in white and red berries (Figure 1(a)). CAT was detected only in red grapes. The levels of stilbenes were about 2-fold lower in Albana than in Lambrusco grapes (Figure 1(b)). Resveratrol (RESV) was detected both in the free and mono-glucosylated forms in all the samples. In particular cis-resveratrol (CRESV) and cis-piceid (CPIC) were measured only in the red grapes (Figure 1(b)). The levels of vanillin (VAN), quercetin (QUERC), rutin (RUT) and myricetin (MYR) were also determined by HPLC-DAD both in white and red berries (data not shown). In particular VAN was present at average levels of 1.5 and 2.0 $\mu \mathrm{mol} / \mathrm{kgFW}$ respectively in white and red berries; QUERC at average levels of 11.9 and $4.9 \mu \mathrm{mol} / \mathrm{kgFW}$; RUT at average levels of 1.8 and $1.0 \mu \mathrm{mol} / \mathrm{kgFW}$ and MYR at average levels of 0.1 and $1.1 \mu \mathrm{mol} / \mathrm{kgFW}$. All the other compounds were not detected either in Albana or Lambrusco samples.

Free biogenic amine levels were determined by HPLC in conventional, organic and biodynamic berries of Albana and Lambrusco varieties (Figure 2). In all the samples putrescine (PUT) was the most abundant polyamine but with a decrease going from conventional to biodynamic samples. Cadaverine (CAD) was totally absent in
Table 1. Total polyphenol, anthocyanin and antioxidant activity levels in Albana and Lambrusco berries grown following conventional (AC and LC), organic (AO and LO) and biodynamic (AB and LB) agricultural practices. Polyphenols are expressed as $g$ of gallic acid (GA) equivalent per kilogram of fresh weight (g GA eq/kgFW); anthocyanins as the variation of absorbance units for kilograms of fresh weight $\left(\Delta_{\mathrm{Abs}} / \mathrm{kgFW}\right)$; antioxidant activity as $\mathrm{g}$ of ascorbic acid (AA) equivalent per kilogram of fresh weight (g AA eq/kgFW). The star symbol indicates the statistically significant differences (Student's $t$-test, $p<0.05$ ) among data of the same group. Data are the mean $\pm \operatorname{SE}(n=4)$.

\begin{tabular}{cccc}
\hline Samples & $\begin{array}{c}\text { Polyphenols } \\
\text { (g GA eq/kgFW) }\end{array}$ & $\begin{array}{c}\text { Anthocyanins } \\
\square\end{array} \Delta_{\text {Abs }}$ kgFW) & $\begin{array}{c}\text { Antioxidant activity } \\
\text { (g AA eq/kgFW) }\end{array}$ \\
\hline AC & $4.7 \pm 0.5$ & $98 \pm 2.5$ & $7.4 \pm 0.5$ \\
AO & $4.2 \pm 0.6$ & ${ }^{*} 84 \pm 5.7$ & ${ }^{*} 5.9 \pm 0.1$ \\
AB & $4.9 \pm 0.7$ & $104 \pm 9.6$ & $7.8 \pm 0.8$ \\
LC & $8.5 \pm 0.5$ & ${ }^{*} 31265 \pm 250.7$ & ${ }^{*} 20.2 \pm 1.0$ \\
LO & $8.3 \pm 0.4$ & $18740 \pm 134.8$ & ${ }^{*} 25.5 \pm 1.0$ \\
LB & $7.8 \pm 0.9$ & $21130 \pm 235.9$ & ${ }^{*} 29.5 \pm 1.3$ \\
\hline
\end{tabular}
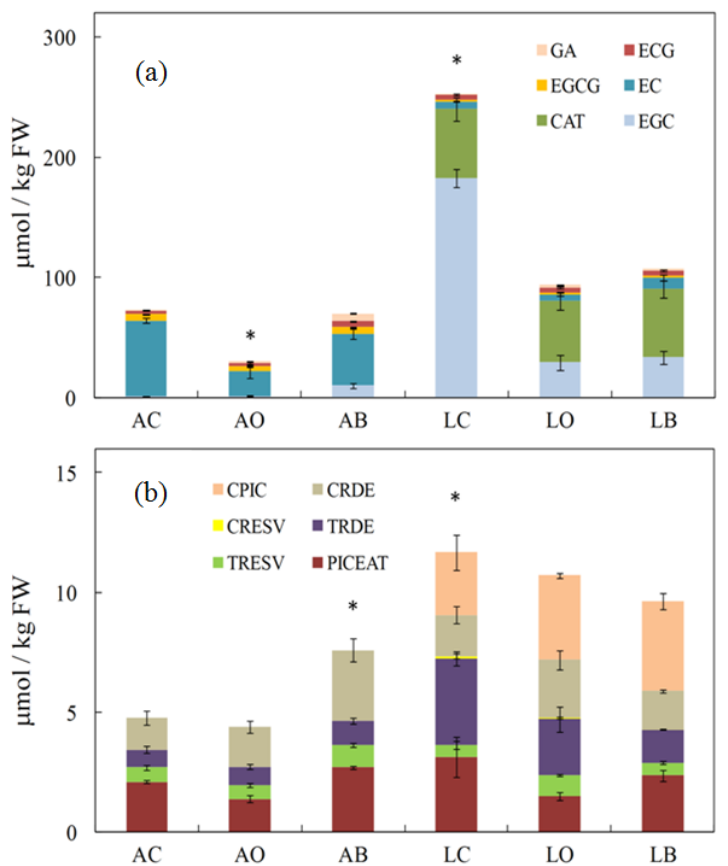

Figure 1. Catechin (a) and stilbene (b) levels ( $\mu \mathrm{mol} / \mathrm{kgFW})$ measured by HPLC-DAD measured in Albana and Lambrusco berries grown following conventional (AC and LC), organic (AO and LO) and biodynamic (AB and $L B)$ agricultural practices. GA, gallic acid; ECG, epicatechingallate; EGCG, epigallocatechin-gallate; EC, epicatechin; CAT, catechin; EGC, epigallocatechin; CRDE, cis-resveratroloside; CPIC, cis-piceid; CRESV, cis-resveratrol; TRDE, trans-resveratroloside TRESV, trans-resveratrol; PICEAT, piceatannol. The star symbol indicates the statistically significant differences (Student's $t$-test, $p<0.05$ ) of the total level of samples of the same group. Data are the mean $\pm S E$ (n=4). 


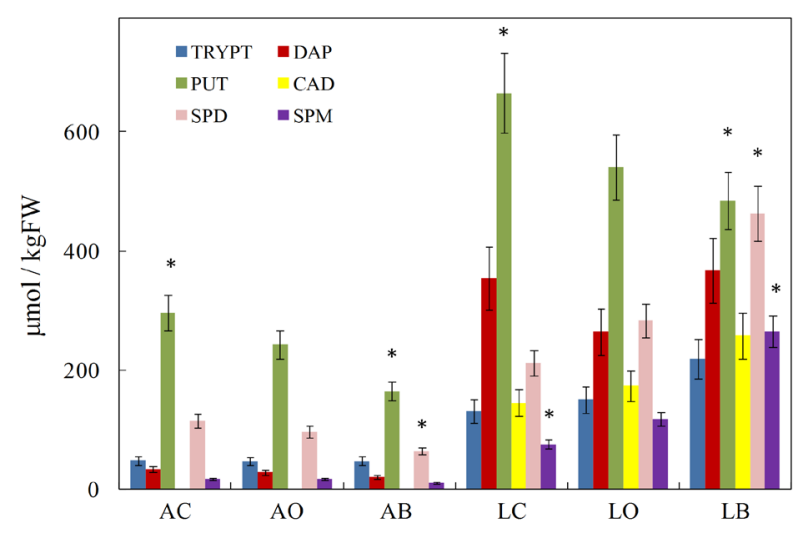

Figure 2. Biogenic amine levels $(\mu \mathrm{mol} / \mathrm{kgFW})$ measured in Albana and Lambrusco berries grown following conventional (AC and LC), organic (AO and LO) and biodynamic (AB and LB) agricultural practices. TRYPT, tryptamine; DAP, diamine-propane; PUT, putrescine; CAD, cadaverine; SPD, spermidine; SPM, spermine. The star symbol indicates the statistically significant differences (Student's $t$-test, $p<0.05$ ) among single compounds of the same group. Data are the mean $\pm \operatorname{SE}(n=4)$.

Albana samples. Among monoamines, only tryptamine (TRYPT) was present and in both grape cultivars averaging 3.5-fold higher in red than in white berries (Figure 2). The total level of amines in red berries was on average 4.2-fold higher than that of white berries independently of the used agricultural method, with respectively $1500 \mu \mathrm{mol} / \mathrm{kgFW}$ for conventional and organic Lambrusco (LC and LO), $2050 \mu \mathrm{mol} / \mathrm{kgFW}$ for biodynamic Lambrusco (LB) and 507, 430 and $306 \mu \mathrm{mol} / \mathrm{kgFW}$ respectively for conventional (AC), organic (AO) and biodynamic (AB) Albana berries.

\subsection{Wines}

The levels of total polyphenols and anthocyanins and of antioxidant activity were determined in the wines produced from conventional, organic and biodynamic Albana and from organic and biodynamic Lambrusco grapes following the respective oenological practices (Table 2). Unfortunately given to production technical problems, it was not possible to collect and analyse the conventional Lambrusco wine. Total polyphenols were on average similar in Lambrusco (2.05 g GA eq/L) and Albana (1.75 g GA eq/L) wines, with AB and LB showing respectively the highest values for red and white samples (Table 2). Anthocyanin content of red wines was on average 11.4-fold higher than that of white wines, with again the highest levels in AB and LB (Table 2). The antioxidant activity measured by DPPH method was on average 1.7-fold higher in red than in white wines (Table 2). Analogously to what showed for berries, this result seems to be in agreement with the levels of total
Table 2. Total polyphenol, anthocyanin and antioxidant activity levels in Albana and Lambrusco wines obtained following conventional (AC and LC), organic (AO and LO) and biodynamic (AB and $\mathrm{LB})$ oenological practices. Polyphenols are expressed as $\mathrm{g}$ of gallic acid (GA) equivalent for litre (g GA eq/L); anthocyanins are expressed as the variation of absorbance units for litre $\left(\Delta_{\mathrm{Abs}} / \mathrm{L}\right)$; antioxidant activity data are expressed as $g$ of ascorbic acid (AA) equivalent for litre (g AA eq/L). The star symbol indicates the statistically significant differences (Student's $t$-test, $p<0.05$ ) among data of the same group. Data are the mean \pm SE (n = 4).

\begin{tabular}{cccc}
\hline Samples & $\begin{array}{c}\text { Polyphenols } \\
\text { (g GA eq/L) }\end{array}$ & $\begin{array}{c}\text { Anthocyanins } \\
\square\left(\boldsymbol{\Delta}_{\mathrm{Abs}} / \mathrm{L}\right)\end{array}$ & $\begin{array}{c}\text { Antioxidant activity } \\
\text { (g AA eq/L) }\end{array}$ \\
\hline AC & $1.67 \pm 0.04$ & ${ }^{*} 80.0 \pm 5.8$ & $2.5 \pm 0.6$ \\
AO & $1.26 \pm 0.08$ & ${ }^{*} 114.1 \pm 9.3$ & ${ }^{*} 1.4 \pm 0.2$ \\
AB & ${ }^{*} 2.33 \pm 0.12$ & ${ }^{*} 243.2 \pm 12.4$ & $2.9 \pm 0.9$ \\
LC & - & - & - \\
LO & $1.76 \pm 0.05$ & $1535.6 \pm 104.5$ & $3.7 \pm 0.4$ \\
LB & ${ }^{*} 2.34 \pm 0.04$ & $1765.8 \pm 155.2$ & $4.0 \pm 0.7$ \\
\hline
\end{tabular}

anthocyanins.

The quantification of catechins by HPLC-DAD, evidenced a similar profile both in white and red wines (Figure 3(a)), with $\mathrm{AB}$ showing the highest level of total catechins (given to the large CAT amount). The AC, LO and LB wines showed similar levels and profiles with both free and mono-glucosylated forms of stilbenes, while in $\mathrm{AO}$ and $\mathrm{AB}$ wines only trans-resveratrol (TRESV), trans-resveratroloside (TRDE) and CPIC, were detected (Figure 3(b)). In all wines CPIC was the most abundant stilbene.

As previously shown for berries, the levels of four other polyphenols were determined by HPLC-DAD (data not shown). In white wines only VAN, QUERC and RUT were detectable, while MYR was only present in red wines. In all wines QUERC was the most abundant compound with maximum amount in AB sample (1932 $\mu \mathrm{mol} / \mathrm{L})$. In general in Albana wines VAN, QUERC and RUT were present at average levels of 460, 305 and 817 $\mu \mathrm{mol} / \mathrm{L}$ while in Lambrusco (organic and biodynamic) wines 143, 204 and $144 \mu \mathrm{mol} / \mathrm{L}$ respectively. In Lambrusco MYR was detectable at an average level of 160 $\mu \mathrm{mol} / \mathrm{L}$. All the other compounds were not detected either in Albana or Lambrusco samples.

The levels of free biogenic amines were determined by HPLC in conventional, organic and biodynamic Albana wines and in organic and biodynamic Lambrusco wines (Figure 4). On average 1.3-fold higher levels of amines were detected in red wines (about $5200 \mu \mathrm{mol} / \mathrm{L}$ ) with respect to white ones (about $4050 \mu \mathrm{mol} / \mathrm{L}$ ). In comparison to grape berries (Figure 2), in wines two additional amines, histamine (HIM) and tyramine (TYM), were detected as a consequence of the microbial fermentation 


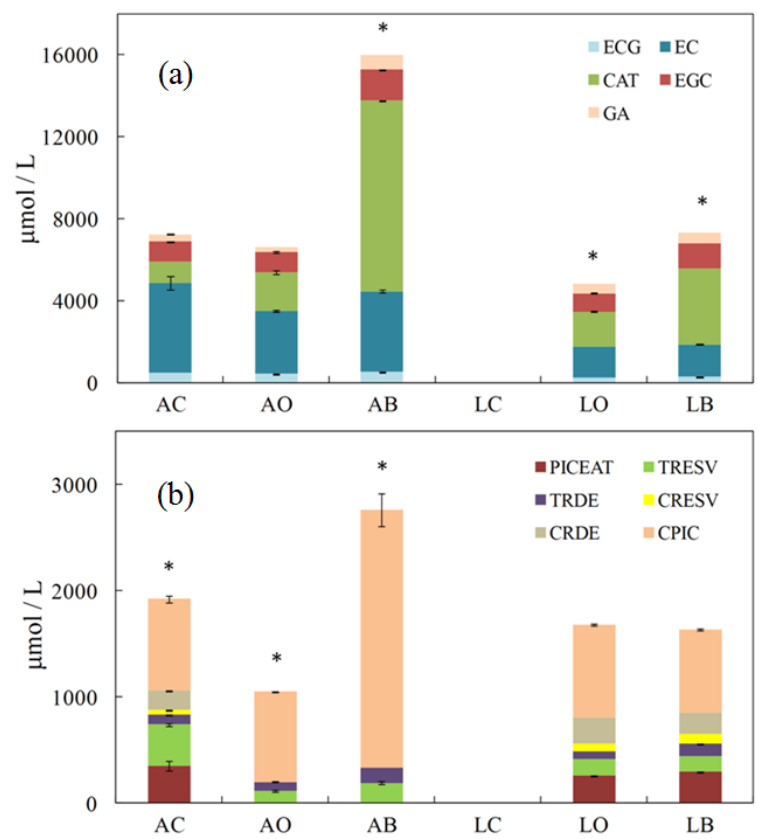

Figure 3. Catechin (a) and stilbene (b) levels ( $\mu \mathrm{mol} / \mathrm{L}$ ) measured by HPLC-DAD in Albana and Lambrusco wines obtained following conventional (AC), organic (AO and LO) and biodynamic (AB and LB) oenological practices. ECG, epicatechin-gallate; EC, epicatechin; CAT, catechin; EGC, epigallocatechin; GA, gallic acid; PICEAT, piceatannol: TRESV, trans-resveratrol; TRDE, trans-resveratroloside; CRESV, cis-resveratrol; CRDE, cis-resveratroloside; CPIC, cis-piceid. The star symbol indicates the statistically significant differences (Student's $t$-test, $p<0.05$ ) of the total level of the samples of the same group. Data are the mean \pm SE $(n=4)$.

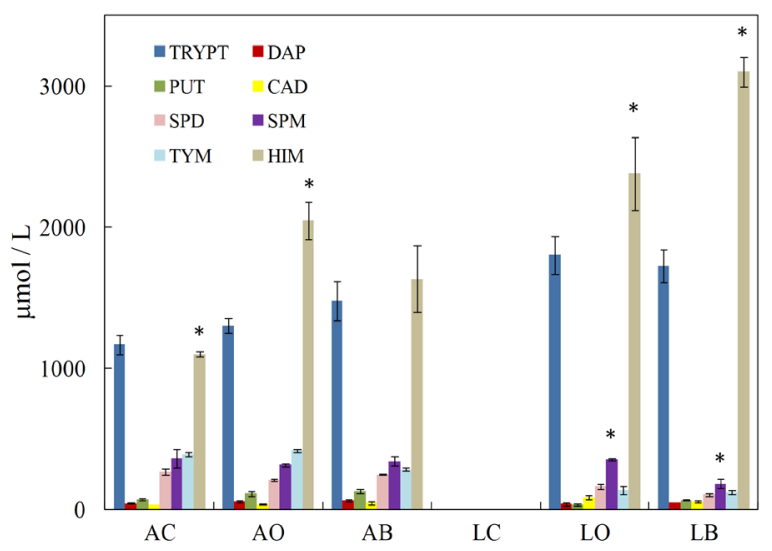

Figure 4. Biogenic amine levels $(\mu \mathrm{mol} / \mathrm{L})$ measured in $\mathrm{Al}$ bana and Lambrusco wines obtained following conventional (AC), organic (AO and LO) and biodynamic (AB and LB) oenological practices. TRYPT, tryptamine; DAP, diaminepropane; PUT, putrescine; CAD, cadaverine; SPD, spermidine; SPM, spermine; TYM, tyramine; HIM, histamine. The star symbol indicates the statistically significant differences (Student's $t$-test, $p<0.05$ ) among single compounds of the same group. Data are the mean \pm SE $(n=4)$. process that occurs during winemaking.

The highest biogenic amine amounts were detected in AO and LB, respectively for white and red wines, this mostly given to the high levels of monoamines (TRYPT, TYM and HYM) which were largely formed after winemaking independently from the adopted oenological methodology. HYM and TRYPT were the most abundant amines both in white and red wines showing average levels respectively of 1.7 and 1.3-fold higher in red than in white wines. On the contrary, TYM was on average 2.6-fold most abundant in Albana than in Lambrusco wines (Figure 4).

\section{Discussion}

The present analytical data regarding white and red grape berries and wines did not generally evidence a significant difference among the samples obtained from different agricultural and winemaking practices (with some exceptions given to single compounds of few samples), while a greater difference, concerning in particular biogenic amine and anthocyanin levels (Tables 1 and 2; Figures 2 and 4), was measured between white and red samples, in accordance with other published papers [7,9,21,22]. Lambrusco berries showed the presence of higher amounts of biogenic amines (Figure 2), of anthocyanins and a higher antioxidant activity (Table 1), with respect to Albana. In both white and red grapes the amine species generally considered most dangerous for human health, namely HIM and TYM, were not detected, while high amounts of PUT were measured in red samples (Figure 2). The HPLC-DAD analyses of catechins and stilbenes evidenced a different spectrum of metabolites between white and red berries with CAT, CRESV and CPIC only present in red berries. The total levels of catechins were similar between the two cultivars (with the exception of LC), while 2-fold higher amounts of stilbenes were detected in red grapes (Figures 1(a) and (b)).

In accordance to grape samples, the amount of biogenic amines was slightly higher in red compared to white wines, which presented large amounts of TRYPT and HIM, while on the contrary TYM was present in higher amounts in white respect to red wines (Figure 4). It has been demonstrated that HIM and TYM may represent potential threats for human health and are mainly produced during winemaking by bacteria of the Lactobacillus or Oenococcus genera which are usually present in the must during the fermentation process [15]. The variability of the biogenic amine contents and/or profiles in wine could be explained on the basis of differences in the geographical region, grape variety, raw material quality, winemaking process, vintage, time and storage conditions and possible microbial contaminations [15]. Cultivar related differences in biogenic amine content have 
been already observed for instance in Spanish [22], Greek [23] and Italian grapes and wines [24]. In this view and in contrast to the present data on Albana and Lambrusco wines, it has recently been demonstrated a major amount of biogenic amines in Italian Pignoletto white wines respect to Sangiovese red wines [7] independently from the adopted oenological practices, leading again to hypothesise that grape variety represents the variable mostly influencing the levels of biogenic amines in the final wine product. Therefore given the large number of factors involved, it seems not easily feasible, even though desirable, to minimise the formation of biogenic amines (in particular of those mostly dangerous for human health) during winemaking. The reduction of amine formation might be partially achievable by carefully selecting the grape variety and optimising the winemaking parameters (such as temperature, maceration time, yeast and used bacterial strains).

Other factors mostly influencing the formation of biogenic amines may also be the levels and metabolic profiles of polyphenols present at first in the grape berries and lately in wines. In fact it has been reported that high concentrations of some phenolic compounds (naturally present mostly in red grapes) affect biogenic amine production by inhibiting lactic acid bacteria growth [25]. Recent data published on levels of polyphenols and biogenic amines in Sangiovese and Pignoletto wines [7] evidenced the presence of different stilbene profiles between the two cultivar. In fact, in white wines only free RESV (both trans and cis) was detected, while in red ones also PICEAT and mono-glucosylated stilbenes (RDE and PIC) were present. PICEAT in particular was demonstrated to have an inhibitory activity on the PUT forming enzyme ornithine decarboxylase (ODC) [26], thus confirming a possible beneficial effect of stilbenes on the decrement of biogenic amines. The authors hypothesised that the presence of PICEAT and mono-glycosilated stilbenes may have partially inhibited the fermenting activity of bacteria present in Sangiovese red must so reducing in the wines the levels of HIM, TYM and PUT, which by contrast were freely synthesised in Pignoletto that in fact showed on average 3.6-fold more biogenic amines [7]. On the contrary to previous data on Pignoletto and Sangiovese, Albana and Lambrusco berries and wines did not evidence a striking difference with regard to profiles and the levels of catechins and stilbenes (Figures 1 and 3), with the presence of PICEAT and mono-glucosylated stilbenes both in white and red samples. As regards biogenic amine levels, only a slight difference was pointed out between Albana and Lambrusco wines (1.3-fold higher in red than in white samples), both showing almost similar amounts of potentially detrimental monoamines and in particular of HIM and TRYPT (Figure 4). The present data may therefore con- firm indirectly the previous formulated hypothesis [7] regarding a possible direct influence of specific stilbene species on the formation of biogenic amines.

In conclusion it is well known that the interaction between different food metabolites (such as biogenic amines and polyphenols) and their relative biosynthetic pathways, may contribute to the healthy or detrimental characteristics of the food itself. Regarding Albana and Lambrusco berries and wines, our data seem to indicate that their metabolic profiles are not particularly influenced by the grape growth conditions or by oenological practices, but mainly by the varietal, physiological and metabolomic characteristics of the food raw material itself.

\section{Acknowledgements}

This work was financed by the project entitled "Valorization of the nutraceutical and healthy properties of grapes and wines grown in the Bologna and Ravenna territory”, Fondazione del Monte di Bologna e Ravenna year 2008 (Bologna, Italy) and from RFO funds from Italian Ministry of Instruction, University and Research (MIUR) to A. Tassoni (2008). We wish to thank all the producers that actively contributed to the collection of the samples.

\section{REFERENCES}

[1] D. K. Asami, Y. J. Hing, D. M. Barret and A. E. Mitchell, "Comparison of the Total Phenolic and Ascorbic Acid Content of Freeze Dried and Air Dried Marionberry, Strawberry and Corn Using Conventional, Organic and Sustainable Agriculture Practices," Journal of Agricultural and Food Chemistry, Vol. 51, No. 5, 2003, pp. 12371241. http://dx.doi.org/10.1021/jf020635c

[2] M. Olsson, C. S. Andersson, S. Oredsson, R. H. Berglund and K. E. Gustavsson, "Antioxidant Levels and Inhibition of Cancer Cell Proliferation in Vitro by Extracts from Organically and Conventionally Cultivated Strawberries," Journal of Agricultural and Food Chemistry, Vol. 54, No. 4, 2006, pp. 1248-1255. http://dx.doi.org/10.1021/jf0524776

[3] J. Kalinova and N. Vrchotova, "The Influence of Organic and Conventional Crop Management, Variety and Year on the Yield and Flavonoid Level in Common Buckwheat Groats,” Food Chemistry, Vol. 127, No. 2, 2011, pp. 602-608.

http://dx.doi.org/10.1016/j.foodchem.2011.01.050

[4] C. Zörb, G. Langenkämper, T. Betsche, K. Niehaus and A. Barsch, "Metabolite Profiling of Wheat Grains (Triticum aestivum L.) from Organic and Conventional Agriculture," Journal of Agricultural and Food Chemistry, Vol. 54, No. 21, 2006, pp. 8301-8306. http://dx.doi.org/10.1021/jf0615451

[5] A. Valavanidis, T. Vlachogianni, A. Psomas, A. Zovoilli 
and V. Siatis, "Polyphenolic Profile and Antioxidant Activity of Five Apple Cultivars Grown under Organic and Conventional Agricultural Practices,” International Journal of Food Science and Technology, Vol. 44, No. 6, 2009, pp. 1167-1175. http://dx.doi.org/10.1111/j.1365-2621.2009.01937.x

[6] J. R. Reeve, L. Carpenter-Boggs, J. P. Reganold, A. L. York, G. McGourthy and L. P. McCloskey, "Soil and Winegrape Quality in Biodynamically and Organically Managed Vineyards," American Journal of Enology and Viticulture, Vol. 56, No. 4, 2005, pp. 367-376.

[7] A. Tassoni, N. Tango and M. Ferri, "Comparison of Biogenic Amine and Polyphenol Profiles of Grape Berries and Wines Obtained Following Conventional, Organic and Biodynamic Agricultural and Oenological Practices,” Food Chemistry, Vol. 139, No. 1, 2013, pp. 405-413. http://dx.doi.org/10.1016/j.foodchem.2013.01.041

[8] I. Urquiaga and F. Leighton, "Wine and Health: Evidence and Mechanisms," In: A. P. Simopoulos, Ed., Nutrition and Fitness: Mental Health, Aging, and the Implementation of a Healthy Diet and Physical Activity Lifestyle, World Reviews of Nutrition and Dietetics, Basel, Karger, Vol. 95, 2005, pp. 122-139. http://dx.doi.org/10.1159/000088299

[9] R. C. Minussi, M. Rossi, L. Bologna, L. Cordi, D. Rotilio, G. M. Pastore and N. Durán, "Phenolic Compounds and Total Antioxidant Potential of Commercial Wines," Food Chemistry, Vol. 82, No. 3, 2003, pp. 409-416. http://dx.doi.org/10.1016/S0308-8146(02)00590-3

[10] L. Bavaresco, C. Fregoni, M. I. van Zeller de Macedo Basto Gançalves and S. Vezzulli, "Physiology and Molecular Biology of Grapevine Stilbenes: An Update,” In: K. A. Roubelakis-Angelakis, Ed., Grapevine Molecule Physiology and Biotechnology, Springer Science, New York, 2009, pp. 341-364. http://dx.doi.org/10.1007/978-90-481-2305-6_12

[11] R. E. King, J. A. Bomser and D. B. Min, "Bioactivity of Resveratrol," Comprehensive Reviews in Food Science and Food Safety, Vol. 5, No. 3, 2006, pp. 65-70. http://dx.doi.org/10.1111/j.1541-4337.2006.00001.x

[12] G. Regev-Shoshani, O. Shoseyov, I. Bilkis and Z. Kerem, "Glycosylation of Resveratrol Protects It from Enzymic Oxidation,” Biochemical Journal, Vol. 374, No. Pt 1, 2003, pp. 157-163.

[13] L. Bavaresco, S. Vezzulli, P. Battilani, P. Giorni, A. Pietri and T. Bertuzzi, "Effect of Ochratoxin A-Producing Aspergilli on Stilbenic Phytoalexin Synthesis in Grapes,” Journal of Agricultural and Food Chemistry, Vol. 51, No. 21, 2003, pp. 6151-6157. http://dx.doi.org/10.1021/jf0301908

[14] T. Kusano, T. Berberich, C. Tateda and Y. Takahashi, "Polyamines: Essential Factors for Growth and Survival," Planta, Vol. 228, No. 3, 2008, pp. 367-381. http://dx.doi.org/10.1007/s00425-008-0772-7

[15] L. Beneduce, A. Romano, A. Capozzi, P. Lucas, L. Barnavon, B. Bach, P. Vuchot, F. Crieco and G. Spano, "Biogenic Amines in Wines," Annals of Microbiology, Vol. 60, No. 4, 2010, pp. 573-578.

\section{http://dx.doi.org/10.1007/s13213-010-0094-4}

[16] EFSA Panel on Biological Hazards, "Scientific Opinion on Risk Based Control of Biogenic Amine Formation in Fermented Foods,” EFSA Journal, Vol. 9, No. 10, 2011, pp. 2393-2486.

[17] V. L. Singleton, R. Orthofer and R. M. Lamuela-Raventos, "Analysis of Total Phenols and Other Oxidation Substrates and Antioxidants by Means of Folin-Ciocalteu Reagent," Methods in Enzymology, Vol. 299, 1999, pp. 152178. http://dx.doi.org/10.1016/S0076-6879(99)99017-1

[18] M. Ferri, A. Tassoni, M. Franceschetti, L. Righetti, M. J. Naldrett and N. Bagni, "Chitosan Treatment Induces Changes of Protein Expression Profile and Stilbene Distribution in Vitis vinifera Cell Suspensions," Proteomics, Vol. 9, No. 3, 2009, pp. 610-624. http://dx.doi.org/10.1002/pmic.200800386

[19] W. Brand-Williams, M. E. Cuvelier and C. Berset, "Use of a Free Radical Method to Evaluate Antioxidant Activity,” Lebensmittel-Wissenschaft und Technologie, Vol. 28, No. 1, 1995, pp. 25-30.

[20] A. Tassoni, M. van Buuren, M. Franceschetti, S. Fornalè and N. Bagni, "Polyamine Content and Metabolism in Arabidopsis thaliana and Effect of Spermidine on Plant Development," Plant Physiology and Biochemistry, Vol. 38, No. 5, 2000, pp. 383-393. http://dx.doi.org/10.1016/S0981-9428(00)00757-9

[21] N. Landrault, P. Poucheret, P. Ravel, F. Gasc, G. Cros and P. L. Teissedre, "Antioxidant Capacities and Phenolics Levels of French Wines from Different Varieties and Vintages," Journal of Agricultural and Food Chemistry, Vol. 49, No. 7, 2001, pp. 3341-3348. http://dx.doi.org/10.1021/jf010128f

[22] J. M. Landete, S. Ferrer, L. Polo and I. Pardo, "Biogenic Amines in Wines from Three Spanish Regions," Journal of Agricultural and Food Chemistry, Vol. 53, No. 4, 2005, pp. 1119-1124. http://dx.doi.org/10.1021/jf049340k

[23] E. H. Soufleros, E. Buoloumpasi, A. Zotou and Z. Loukou, "Determination of Biogenic Amines in Greek Wines by HPLC and Ultraviolet Detection after Dansylation and Examination of Factors Affecting Their Presence and Concentration,” Food Chemistry, Vol. 101, No. 2, 2007, pp. 704-716.

http://dx.doi.org/10.1016/j.foodchem.2006.02.028

[24] V. Del Prete, A. Costantini, F. Cecchini, M. Morassut and E. Garcia-Moruno, "Occurrence of Biogenic Amines in Wine: The Role of Grapes,” Food Chemistry, Vol. 112, No. 2, 2009, pp. 474-481. http://dx.doi.org/10.1016/j.foodchem.2008.05.102

[25] M. R. Alberto, M. E. Arena and M. C. Manca de Nadra, "Putrescine Production from Agmatine by Lactobacillus hilgardii: Effect of Phenolic Compounds," Food Control, Vol. 18, No. 8, 2007, pp. 898-903. http://dx.doi.org/10.1016/j.foodcont.2006.05.006

[26] F. Wolter, S. Ulrich and J. Stein, "Molecular Mechanisms of the Chemopreventive Effects of Resveratrol and Its Analogs in Colorectal Cancer: Key Role of Polyamines?” Journal of Nutrition, Vol. 134, No. 12, 2004, pp. 32193222. 


\section{Abbreviations}

AA: ascorbic acid;

CAD: cadaverine;

CAT: catechin;

CPIC: cis-piceid;

CRDE: cis-resveratroloside;

CRESV: cis-resveratrol;

DAD: diode array detector;

DPPH: 2,2-diphenyl-1-picrylhydrazyl;

EC: epicatechin;

ECG: epicatechin-gallate;

EGC: epigallocatechin;

EGCG: epigallocatechin-gallate;

GA: gallic acid;
HIM: histamine

MYR: myricetin;

PICEAT: piceatannol;

PUT: putrescine;

QUERC: quercetin;

RUT: rutin;

SPD: spermidine;

SPM: spermine;

TRDE: trans-resveratroloside;

TRESV: trans-resveratrol;

TRYPT: tryptamine;

TYM: tyramine;

VAN: vanillin. 